\title{
EXISTENCE AND UNIQUENESS RESULTS, THE MARKOVIAN PROPERTY OF SOLUTION FOR A NEUTRAL DELAY STOCHASTIC REACTION-DIFFUSION EQUATION IN ENTIRE SPACE
}

\author{
K.K. KENZHEBAEV ${ }^{1}$, A.N. STANZHYTSKYI ${ }^{2}$, AND A.O. TSUKANOVA ${ }^{3}$ \\ ${ }^{1}$ K. Zhubanov Aktobe Regional State University \\ 34 A. Moldagulova St., Aktobe, 030000, KAZAKHSTAN \\ ${ }^{2}$ Taras Shevchenko National University of Kiev \\ 4-E Academician Glushkov St., Kiev, 03127, UKRAINE \\ ${ }^{2,3}$ National Technical University of Ukraine \\ "Igor Sikorsky Kiev Polytechnic Institute" \\ 37 Pobedy St., Kiev, 03056, UKRAINE
}

\begin{abstract}
We establish results, concerning existence, uniqueness, and continuous dependence on an initial datum of a mild solution for neutral stochastic integro-differential equations with variable time delay of reaction-diffusion type. We also establish the Markovian property of this solution. Herewith our emphasis is on unbounded domain $\left\{x \in \mathbb{R}^{d}\right\}$.
\end{abstract}

AMS Subject Classification: 34K40, 34K50, 60H15

Key Words: stochastic differential equation, mild solution, Wiener process, semigroup, Hilbert space

Received: February 27, 2017; Revised: October 1, 2018; Published (online): October 3, 2018 doi: $10.12732 /$ dsa.v28i1.2 Dynamic Publishers, Inc., Acad. Publishers, Ltd. https://acadsol.eu/dsa

\section{INTRODUCTION}

In the given paper we study existence, uniqueness, continuous dependence on an initial datum, and the Markovian property of a solution to the following Cauchy problem for 
a partial stochastic integro-differential equation of reaction-diffusion type with delay

$$
\begin{gathered}
d\left(u(t, x)+\int_{\mathbb{R}^{d}} b(t, x, u(\alpha(t), \xi), \xi) d \xi\right)=\left(\Delta_{x} u(t, x)+f(t, u(\alpha(t), x), x)\right) d t \\
+\sigma(t, u(\alpha(t), x), x) d W(t, x), 0<t \leq T, x \in \mathbb{R}^{d} \\
u(t, x)=\phi(t, x),-r \leq t \leq 0, x \in \mathbb{R}^{d}, r>0,
\end{gathered}
$$

where $\Delta_{x} \equiv \sum_{i=1}^{d} \partial_{x_{i}}^{2}$ is $d$-measurable Laplace operator, $\partial_{x_{i}}^{2} \equiv \frac{\partial^{2}}{\partial x_{i}^{2}}, i \in\{1, \ldots, d\}$, $W(t, x), x \in \mathbb{R}^{d}$, is $L_{2}\left(\mathbb{R}^{d}\right)$-valued $Q$-Wiener process, $\{f, \sigma\}:[0, T] \times \mathbb{R} \times \mathbb{R}^{d} \rightarrow \mathbb{R}$ and $b:[0, T] \times \mathbb{R}^{d} \times \mathbb{R} \times \mathbb{R}^{d} \rightarrow \mathbb{R}$ are some given functions to be specified later, $\phi:[-r, 0] \times \mathbb{R}^{d} \times \Omega \rightarrow \mathbb{R}$ is an initial datum function and $\alpha:[0, T] \rightarrow[-r, \infty)$ is a delay function.

Differential equations with delay have first appeared as mathematical models of real processes with evolution that depends on previous states. Number of works are devoted to intensive study of various aspects from this theory. Let note for this reason [8] with an extensive bibliography. A qualitative theory of stochastic differential equations with delay in finite-dimensional spaces has been investigated intensively. For instance, [9] is dedicated to study of questions on existence of solutions and invariant measures, related with them; stochastic stability and various applications of stochastic equations with delay have been studied in [10]; a systematic investigation of various qualitative questions for a stochastic functional differential equation is given in [2]; issues of optimal control for such a class of equations have been studied in [11].

Neutral stochastic functional differential equations have first appeared in [12]. This work is devoted to investigation of existence and stability of solutions, as well as their controllability. Asymptotic behavior and exponential stability of solutions to equations of this type have been considered in [15] - [17]. Concerning such equations in infinite-dimensional spaces, let remark [14], where a theorem on existence and uniqueness of a mild solution to the Cauchy problem for a neutral stochastic differential equation in a Hilbert space has been proved. But conditions of this theorem are formulated in an abstract form, therefore it is difficult to check them directly for concrete equations in specific spaces, e.g., for stochastic partial differential equations of reaction-diffusion type. For these equations abstract mappings are generated by real-valued functions as the Nemytsky operator. Thus our expectations to obtain the conditions in terms of the coefficients of these equations, i.e. in terms of real-valued functions, are natural. If such conditions are found, it will be possible to check them easily while solving concrete applied problems.

The aim of the paper is to obtain coefficient conditions on existence and uniqueness of a mild solution to $(1)-\left(1^{*}\right)$. This problem is a special case of the problem from [14]. Equation (1) has an applied importance: it models behavior of various dynamical 
systems in physics and mathematical biology. For instance, it describes a well-known Hodgkin-Huxley model in neurophysiology (where $u$ is an electric potential on nerve cells [18]), as well as the Dawson and Fleming models of population genetics [5] (where $u$ is a mass distribution of population).

Let remark that presence of an integral term in (1) turns this equation into a nonlocal neutral stochastic equation of reaction-diffusion type. Nonlocal deterministic equations of this type are well known in literature and have wide range of applications. In particularly, these equations are used in modeling of phytoplankton growth in [6]. They also model distant interactions in epidemic models (see, e.g., [1]) and nonlocal consumption of resources, as well as nonlocal Fisher-KPP equation (see [19] and references therein).

Let note that problem $(1)-\left(1^{*}\right)$ is considered in an unbounded domain $G=\mathbb{R}^{d}$. The principal difference of this problem from the problem in a bounded domain is that a semigroup $\{S(t)\}$, generated by the Laplace operator in a bounded domain $G$ with trivial Dirichlet boundary condition, possesses the exponential contraction property, that is

$$
\|(S(t) u(\cdot))(x)\|_{L_{2}(G)}^{2} \leq M \exp \{-\varpi t\}\|u(x)\|_{L_{2}(G)}^{2}
$$

with $M$ and $\varpi$ positive numbers. It is well known that if $G=\mathbb{R}^{d}$, then (2) is not valid.

This paper is organised as follows. Firstly, we introduce a few necessary results on a solution to the Cauchy problem for a heat equation, needed in what follows. These preliminary results are gathered in section 2 . Then, in section 3 , we introduce a statement of the problem and formulate our main results. Section 4 is devoted to their proof. In the appendix we give the proof of proposition 3.

\section{PRELIMINARIES}

In this section we recall few facts and introduce some notation, needed hereinafter in order to establish our results.

Throughout the paper $H=L_{2}\left(\mathbb{R}^{d}\right)$ will note a real Hilbert space with the inner product $(f, g)_{H}=\int_{\mathbb{R}^{d}} f(x) g(x) d x$ and the associated norm $\|f\|_{H}=\sqrt{\int_{\mathbb{R}^{d}} f^{2}(x) d x}$.

Let $\left\{e_{n}(x), n \in\{1,2, \ldots\}\right\}$ be an orthonormal basis on $H$ such that

$$
\sup _{n \in\{1,2, \ldots\}}\left\|e_{n}\right\|_{L_{\infty}\left(\mathbb{R}^{d}\right)} \leq 1
$$

Let $(\Omega, \mathcal{F}, \mathbf{P})$ be a complete probability space. We now define a $Q$-Wiener $H$-valued 
process $W(t)=W(t, \cdot)$ as follows

$$
W(t, x)=\sum_{n=1}^{\infty} \sqrt{\lambda_{n}} e_{n}(x) \beta_{n}(t), t \geq 0, x \in \mathbb{R}^{d},
$$

where $\left\{\beta_{n}(t), n \in\{1,2, \ldots\}\right\} \subset \mathbb{R}$ are independent standard one-dimensional Brownian motions on $t \geq 0,\left\{\lambda_{n}, n \in\{1,2, \ldots\}\right\}$ is a sequence of positive numbers such that $\lambda=\sum_{n=1}^{\infty} \lambda_{n}<\infty$. Let $\left\{\mathcal{F}_{t}, t \geq 0\right\}$ be a normal filtration on $\mathcal{F}$. We assume that

1) $W(t), t \geq 0$, is $\mathcal{F}_{t}$-measurable;

2) the increments $W(t+h)-W(t)$ are independent of $\mathcal{F}_{t}$ for all $h>0$ and $t \geq 0$.

Note that if $Q: H \rightarrow H$ is a linear continuous operator such that for any $z \in H$

$$
Q z=\sum_{n=1}^{\infty} \lambda_{n}\left(z, e_{n}\right)_{H} e_{n}
$$

then $Q$ is a nuclear nonnegative operator such that

$$
Q e_{n}=\lambda_{n} e_{n}
$$

We set $L_{2}^{0}=L_{2}\left(Q^{\frac{1}{2}} H, H\right)$ - the space of all Hilbert-Schmidt operators, acting from $Q^{\frac{1}{2}} H$ into $H$, equipped with the inner product $(\Psi, \Phi)_{L_{2}^{0}}=\operatorname{Tr}\left[\Psi Q \Phi^{*}\right]$.

Hereafter we will need some results of independent interest from the theory of heat for the following Cauchy problem

$$
\begin{gathered}
\partial_{t} u(t, x)=\Delta_{x} u(t, x), t>0, x \in \mathbb{R}^{d}, \\
u(0, x)=g(x), x \in \mathbb{R}^{d} .
\end{gathered}
$$

Let denote the fundamental solution of the heat equation by

$$
\mathcal{K}(t, x)=\left\{\begin{aligned}
\frac{1}{(4 \pi t)^{\frac{d}{2}}} \exp \left\{-\frac{|x|^{2}}{4 t}\right\}, & t>0, \quad x \in \mathbb{R}^{d}, \\
0, & t<0, \quad x \in \mathbb{R}^{d} .
\end{aligned}\right.
$$

Proposition 1. [20, XIV, Theorem 2] If $g$ in $\left(4^{*}\right)$ belongs to $H$, then a solution of (4) $-\left(4^{*}\right)$ is given by the following formula

$$
u(t, x)=\int_{\mathbb{R}^{d}} \mathcal{K}(t, x-\xi) g(\xi) d \xi
$$

an besides $u$ belongs to $C^{\infty}\left((0, \infty) \times \mathbb{R}^{d}\right)$. 
Proposition 2. [20, $p$. 242 - 244] Operators $S(t): H \rightarrow H$, generating a solution of the Cauchy problem $(4)-\left(4^{*}\right)$ by the rule

$$
u(t, x)=(S(t) g(\cdot))(x)=\int_{\mathbb{R}^{d}} \mathcal{K}(t, x-\xi) g(\xi) d \xi,
$$

form a contraction $\left(C_{0}\right)$-semigroup of operators, i.e. the following estimate is valid

$$
\|(S(t) g(\cdot))(x)\|_{H}^{2} \leq\|g(x)\|_{H}^{2},
$$

and besides the Laplacian $\Delta_{x}$ is the infinitesimal generator of this semigroup.

The next result is a sort of analogue for [3, proposition A.1.1, p. 307]. But, in contrast with this work, we do not require analyticity of the semigroup $\{S(t), t \geq 0\}$ and the property of exponential decreasing

$$
\|S(t)\|_{H}^{2} \leq M \exp \{-\varpi t\}, t \geq 0
$$

with $M$ and $\varpi$ positive numbers.

Let $A: D(A) \in H \rightarrow H$ be the infinitesimal generator of the contraction semigroup $\{S(t), t \geq 0\}$ on $H$. Similarly to [3], for any $0<\gamma<1$ and $p>1$ we define a linear bounded operator $R_{\gamma}$ on $L_{p}([0, T], H)$ by the rule

$$
R_{\gamma} \varphi(t)=\int_{0}^{t}(t-s)^{\gamma-1} S(t-s) \varphi(s) d s, \varphi \in L_{p}([0, T], H) .
$$

The proof of the next proposition will be given in the appendix.

Proposition 3. If $\gamma>\frac{1}{p}$, then $R_{\gamma}$ is a bounded operator, acting from $L_{p}([0, T], H)$ to $C([0, T], H)$.

Proposition 4. [13, p. 274] For partial derivatives of $\mathcal{K}$ the following estimate is true

$$
\left|\partial_{t}^{r} \partial_{x}^{s} \mathcal{K}(t, x)\right| \leq c_{r, s} t^{-\frac{d}{2}-r-\frac{s}{2}} \exp \left\{-\frac{c_{0}|x|^{2}}{t}\right\}
$$

where $c_{r, s}$ is a positive number and $c_{0}<\frac{1}{4}$.

Proposition 5. If $g$ in $\left(4^{*}\right)$ belongs to $L_{1}\left(\mathbb{R}^{d}\right) \cap H$, then a solution of $(4)-\left(4^{*}\right)$ satisfies the following limit relations

$$
\lim _{|x| \rightarrow \infty} u(t, x)=0, \quad \lim _{|x| \rightarrow \infty} \partial_{t} u(t, x)=0
$$


Proof. The proof follows from the standard convergence theorem on the passage to the limit under an integral sign and Lebesgue's differentiation theorem on differentiability of the Lebesgue integral with respect to a parameter, with the use of estimate (7).

From propositions 1 and 5 we obtain the following result.

Proposition 6. [7, p. 360] If relations (8) are valid, then a solution of $(4)-\left(4^{*}\right)$ satisfies the estimate

$$
\begin{aligned}
& \sup _{0 \leq t \leq T} \int_{\mathbb{R}^{d}}\left(\Delta_{x} u(t, x)\right)^{2} d x=\sup _{0 \leq t \leq T} \int_{\mathbb{R}^{d}}\left\|D_{x}^{2} u(t, x)\right\|_{d}^{2} d x \\
& \quad \leq C_{T} \int_{\mathbb{R}^{d}}\left\|D_{x}^{2} g(x)\right\|_{d}^{2} d x
\end{aligned}
$$

for some $C_{T}>0$, depending only on $T$, where $\nabla_{x} \equiv\left(\partial_{x_{1}} \ldots \partial_{x_{d}}\right)^{\mathrm{T}}$ is the gradientvector, $D_{x}^{2} \equiv\left(\begin{array}{ccc}\partial_{x_{1}}^{2} & \ldots & \partial_{x_{1} x_{d}} \\ \vdots & \ddots & \vdots \\ \partial_{x_{d} x_{1}} & \ldots & \partial_{x_{d}}^{2}\end{array}\right)$ is the Hessian matrix, $\|\cdot\|_{d}$ is the corresponding norm of matrix.

\section{FORMULATION OF THE PROBLEM}

In the article we impose the following conditions

(1) $\alpha:[0, T] \rightarrow[-r, \infty)$ is a continuous function such that $\alpha(t) \leq t, 0 \leq t \leq T$.

(2) $\{f, \sigma\}:[0, T] \times \mathbb{R} \times \mathbb{R}^{d} \rightarrow \mathbb{R}, b:[0, T] \times \mathbb{R}^{d} \times \mathbb{R} \times \mathbb{R}^{d} \rightarrow \mathbb{R}$ are measurable with respect to all of their variables functions, $b$ is continuous in the first argument.

(3) The initial datum function $\phi(t, \cdot, \omega):[-r, 0] \times \Omega \rightarrow H$ is $\mathcal{F}_{0}$-measurable random variable with almost surely continuous paths and such that

$$
\mathbf{E} \sup _{-r \leq t \leq 0}\|\phi(t, \cdot)\|_{H}^{p}<\infty, p>2 .
$$

(4) There exists a constant $L>0$ and a function $\chi:[0, T] \times \mathbb{R}^{d} \rightarrow$

$\rightarrow[0, \infty)$ with

$$
\sup _{0 \leq t \leq T} \int_{\mathbb{R}^{d}} \chi^{2}(t, x) d x<\infty
$$


such that the following linear-growth and Lipschitz conditions are valid for $f$ and $\sigma$

$$
\begin{gathered}
|f(t, u, x)| \leq \chi(t, x)+L|u|, \\
0 \leq t \leq T, u \in \mathbb{R}, x \in \mathbb{R}^{d}, \\
|f(t, u, x)-f(t, v, x)| \leq L|u-v|, \\
0 \leq t \leq T,\{u, v\} \subset \mathbb{R}, x \in \mathbb{R}^{d}, \\
\sigma^{2}(t, u, x) \leq L^{2}\left(1+u^{2}\right), \\
0 \leq t \leq T, u \in \mathbb{R}, x \in \mathbb{R}^{d}, \\
|\sigma(t, u, x)-\sigma(t, v, x)| \leq L|u-v|, \\
0 \leq t \leq T,\{u, v\} \subset \mathbb{R}, x \in \mathbb{R}^{d} .
\end{gathered}
$$

(5) There exists a function $b_{1}: \mathbb{R}^{d} \times \mathbb{R}^{d} \rightarrow[0, \infty)$, satisfying the following conditions

$$
\begin{gathered}
\int_{\mathbb{R}^{d}} \int_{\mathbb{R}^{d}} b_{1}(x, \xi) d \xi d x<\infty, \\
\int_{\mathbb{R}^{d}}\left(\int_{\mathbb{R}^{d}} b_{1}(x, \xi) d \xi\right)^{2} d x<\infty,
\end{gathered}
$$

such that

$$
|b(t, x, 0, \xi)| \leq b_{1}(x, \xi), 0 \leq t \leq T,\{x, \xi\} \subset \mathbb{R}^{d} .
$$

(6) there exists a function $l: \mathbb{R}^{d} \times \mathbb{R}^{d} \rightarrow[0, \infty)$ such that

$$
\begin{gathered}
|b(t, x, u, \xi)-b(t, x, v, \xi)| \leq l(x, \xi)|u-v|, \\
0 \leq t \leq T,\{x, \xi\} \subset \mathbb{R}^{d},\{u, v\} \subset \mathbb{R}
\end{gathered}
$$

where $l$ satisfies the following conditions

$$
\begin{gathered}
\int_{\mathbb{R}^{d}} \sqrt{\int_{\mathbb{R}^{d}} l^{2}(x, \xi) d \xi} d x<\infty, \\
\int_{\mathbb{R}^{d}} \int_{\mathbb{R}^{d}} l^{2}(x, \xi) d \xi d x<\infty .
\end{gathered}
$$

(7) For any $x \in \mathbb{R}^{d}$ there are derivatives $\partial_{x} b, D_{x}^{2} b$, and for $\nabla_{x} b$ and $D_{x}^{2} b$ the following linear-growth condition with respect to the third argument is true

$$
\begin{gathered}
\left|\nabla_{x} b(t, x, u, \xi)\right|+\left\|D_{x}^{2} b(t, x, u, \xi)\right\|_{d} \leq \psi(t, x, \xi)(1+|u|) \\
0 \leq t \leq T,\{x, \xi\} \subset \mathbb{R}^{d}, u \in \mathbb{R}
\end{gathered}
$$


for $D_{x}^{2} b$ - the following Lipschitz condition

$$
\begin{gathered}
\left\|D_{x}^{2} b(t, x, u, \xi)-D_{x}^{2} b(t, x, v, \xi)\right\|_{d} \leq \psi(t, x, \xi)|u-v|, \\
0 \leq t \leq T,\{x, \xi\} \subset \mathbb{R}^{d},\{u, v\} \subset \mathbb{R}
\end{gathered}
$$

where $\psi:[0, T] \times \mathbb{R}^{d} \times \mathbb{R}^{d} \rightarrow[0, \infty)$ is such that

$$
\begin{gathered}
\sup _{0 \leq t \leq T} \int_{\mathbb{R}^{d}}\left(\int_{\mathbb{R}^{d}} \psi(t, x, \xi) d \xi\right)^{2} d x<\infty, \\
\sup _{0 \leq t \leq T} \iint_{\mathbb{R}^{d}} \psi_{\mathbb{R}^{d}}(t, x, \xi) d \xi d x<\infty
\end{gathered}
$$

and besides for any point $x_{0} \in \mathbb{R}^{d}$ there is its neighborhood $B_{\delta}\left(x_{0}\right)$ and a nonnegative function $\varphi$ such that

$$
\begin{gathered}
\sup _{0 \leq t \leq T} \varphi\left(t, \cdot, x_{0}, \delta\right) \in L_{1}\left(\mathbb{R}^{d}\right) \cap H, \delta>0, \\
\left|\psi(t, x, \xi)-\psi\left(t, x_{0}, \xi\right)\right| \leq \varphi\left(t, \xi, x_{0}, \delta\right)\left|x-x_{0}\right|, \\
0 \leq t \leq T,\left|x-x_{0}\right|<\delta, \xi \in \mathbb{R}^{d} .
\end{gathered}
$$

Definition 7. A continuous random process $u(t, \cdot, \omega):[-r, T] \times$ $\times \Omega \rightarrow H$ is called a mild solution to $(1)-\left(1^{*}\right)$ provided

(1) it is $\mathcal{F}_{t}$-measurable for all $-r \leq t \leq T$;

(2) it satisfies the following integral equation

$$
\begin{aligned}
u(t, \cdot) & =S(t)\left(\phi(0, \cdot)+\int_{\mathbb{R}^{d}} b(0, \cdot, \phi(-r, \zeta), \zeta) d \zeta\right) \\
& -\int_{\mathbb{R}^{d}} b(t, \cdot, u(\alpha(t), \xi), \xi) d \xi \\
& -\int_{0}^{t} \Delta_{(\cdot)}\left(S(t-s) \int_{\mathbb{R}^{d}} b(s, \cdot, u(\alpha(s), \zeta), \zeta) d \zeta\right) d s \\
& +\int_{0}^{t} S(t-s) f(s, u(\alpha(s), \cdot), \cdot) d s \\
+ & \int_{0}^{t} S(t-s) \sigma(s, u(\alpha(s), \cdot), \cdot) d W(s), 0 \leq t \leq T, \\
& u(t, \cdot)=\phi(t, \cdot),-r \leq t \leq 0, r>0 .
\end{aligned}
$$


Remark 8. It is assumed in the definition above that all the integrals from (24) are well defined.

Our first result is concerned with existence and uniqueness of the solution to (1) $-\left(1^{*}\right)$.

Theorem 9 (existence and uniqueness). Suppose assumptions (1) - (7) are satisfied. Then, if

$$
\left(\int_{\mathbb{R}^{d}} \int_{\mathbb{R}^{d}} l^{2}(x, \xi) d \xi d x\right)^{\frac{p}{2}}<\frac{1}{4^{p-1}},
$$

the Cauchy problem (1) $-\left(1^{*}\right)$ has a unique for $0 \leq t \leq T$ mild solution $u(t, \cdot)$. This solution satisfies the condition

$\mathbf{E} \sup _{-r \leq t \leq T}\|u(t, \cdot)\|_{H}^{p}<\infty$

From now on $u(t, x, \phi)$ will denote the solution of $(1)$, satisfying $\left(1^{*}\right)$. The next result is concerned with continuous dependence of $u$ on the corresponding initial datum function $\phi$.

Theorem 10 (continuous dependence). Under the conditions of theorem 9 there exists $C(T)>0$ such that for arbitrary admissible initial datum functions $\phi$ and $\phi_{1}$ the following estimate holds

$$
\begin{aligned}
& \mathbf{E} \sup _{-r \leq t \leq T}\left\|u(t, \cdot, \phi)-u\left(t, \cdot, \phi_{1}\right)\right\|_{H}^{p} \\
& \quad \leq C(T) \mathbf{E} \sup _{-r \leq t \leq 0}\left\|\phi(t, \cdot)-\phi_{1}(t, \cdot)\right\|_{H}^{p}, p>2 .
\end{aligned}
$$

With regard to extension of the mild solution for $(1)-\left(1^{*}\right)$ to the whole semi-axis $t \geq 0$, the following corollary is true.

Corollary 11. If in theorem 9 the conditions from (4) - (7) are valid for $t \geq 0$, then the Cauchy problem (1) - ( $\left.1^{*}\right)$ has a unique mild solution for $t \geq 0$.

If we replace the initial range $[-r, 0]$ from $\left(1^{*}\right)$ by $[s-r, s]$ for arbitrary $0 \leq s \leq t$, it will be possible to guarantee existence and uniqueness of the mild solution to (1) - $\left(1^{*}\right)$ for $0 \leq s \leq t$ with $\mathcal{F}_{s}$-measurable initial datum function $\phi(t, \cdot)$, satisfying assumption (3) for $s-r \leq t \leq s$. From now on such a solution will be denoted by $u(t, s, \cdot, \phi)$. Consequently, if we define $\mathcal{F}_{s}$-measurable initial datum function $\phi(s+$ $\theta, \cdot, \omega) \in H, \theta \in[-r, 0]$, satisfying the conditions from assumption (3), then $u(s+$ $\theta, s, \cdot, \phi)=\phi(s+\theta, \cdot)$ and $u(t, s, \cdot, \phi)$ satisfies $(24)$ for $t \geq s$.

Let $\mathfrak{C}=C([-r, 0], H)$ be the Banach space of all continuous functions, acting from 
$[-r, 0]$ to $H$, equipped with the norm

$$
\|u\|_{\mathfrak{C}}=\sup _{-r \leq t \leq 0}\|u(t, \cdot)\|_{H}=\sup _{-r \leq t \leq 0} \sqrt{\int_{\mathbb{R}^{d}} u^{2}(t, x) d x} .
$$

By $u_{t}(s, \cdot, \phi)=u(t+\theta, s, \cdot, \phi),-r \leq \theta \leq 0$, we denote a shift of the solution $u$ such that $u_{s}(s, \cdot, \phi)=u(s+\theta, s, \cdot, \phi)=\phi(s+\theta, \cdot)$.

Let define by $\mathfrak{C}$, similarly to the finite-dimensional case from [2], the family $\left\{U_{s}^{t}, 0 \leq s \leq t\right\}$ of the operators $U_{s}^{t} \phi \equiv$

$\equiv u(t+\theta, s, \cdot, \phi)$. This family is said to be a family of shift-operators along solutions to $(1)-\left(1^{*}\right)$. It follows from theorem 9 that for any $s$ and $t$ such that $0 \leq s \leq t$ the operator $U_{s}^{t}$ corresponds to every non-random function $\varphi \in \mathfrak{C} \mathfrak{C}$-valued $\mathcal{F}_{s}^{t}(d W)$-measurable random variable $u_{t}(s, \cdot, \varphi)$. Here $\mathcal{F}_{s}^{t}(d W)$ is the minimal $\sigma$-algebra such that the increments $W(\tau)-W(s), s \leq \tau \leq t$, are measurable with respect to it. It is clear that $u_{t}(s, \cdot, \varphi)$ does not depend on $\mathcal{G}_{t}-\sigma$-algebra, generated by the increments $W(s)-W(t), s \geq t$.

From theorem 9 we evidently get the following proposition.

Proposition 12. The family $\left\{U_{s}^{t}, 0 \leq s \leq t\right\}$ of shift-operators has the following evolution property

$$
U_{\tau}^{t} U_{s}^{\tau} \varphi=U_{s}^{t} \varphi, \varphi \in \mathfrak{C},
$$

for every $\tau$ such that $0 \leq s \leq \tau \leq t$.

Let $\mathfrak{D}$ be $\sigma$-algebra of Borel subsets from $\mathfrak{C}$. If for any set $A \in \mathfrak{D}$ we define

$$
\mu_{t}(A)=\mathbf{P}\left\{u_{t}(s, \cdot, \varphi) \in A\right\}=\mathbf{P}\left\{U_{s}^{t} \varphi \in A\right\}=\mathbf{P}\{s, \varphi, t, A\}
$$

then $u_{t}(s, \cdot, \varphi)$ naturally defines a measure on $\mathfrak{D}$. Function $(27)$ is said to be a transition function, corresponding to the random process $u_{t}(s, \cdot, \varphi), s \leq t$. Similarly to the finite-dimensional case from [2], it is possible to show that this function possess all standard properties of the transition probability. The following theorem is valid.

Theorem 13 (the Markovian property). Under the assumptions of theorem 9 the process $u_{t}(s, \cdot, \phi)$ ( $\phi$ satisfies assumption $\left.(3)\right), t \geq s$, is the Markov process on $\mathfrak{C}$ with the transition function, defined by (27).

We will denote by $\mathfrak{B}_{b}(\mathfrak{C})$ the Banach space of all real bounded Borel functions, defined on $\mathfrak{C}$, endowed with sup-norm, by $\mathfrak{C}_{b}(\mathfrak{C})$ - the Banach space of all real bounded continuous functions, defined on $\mathfrak{C}$. If $f \in \mathfrak{B}_{b}(\mathfrak{C})$, then for $0 \leq s \leq t \leq T$ and $\varphi \in \mathfrak{C}$ we define the family of operators

$$
P_{s, t} f(\varphi)=\mathbf{E} f\left(U_{s}^{t} \varphi\right)=\mathbf{E} f\left(u_{t}(s, \cdot, \varphi)\right)
$$


If $P_{s, t} f(\cdot)$ is bounded and continuous for any $f \in \mathfrak{C}_{b}(\mathfrak{C})$, then it will be said that $P_{s, t}$ possess the Feller property. From theorem 10 we have the following corollary.

Corollary 14 (the Feller property). Under the assumptions of theorem 10 the family $P_{s, t}$ from (28) possess the Feller property.

\section{PROOF OF THE THEOREMS AND THEIR COROLLARIES}

\subsection{PROOF OF THEOREM 9 AND ITS COROLLARY 11}

Let consider the Banach space $\mathfrak{B}_{p, T}$ of all $H$-valued $\mathcal{F}_{t}$-measurable random processes $z$ that are continuous in $-r \leq t \leq T$ for almost all fixed $\omega \in \Omega$, endowed with the $\operatorname{norm}\|z(t)\|_{\mathfrak{B}_{p, T}}=$

$=\sqrt[p]{\mathbf{E} \sup _{0 \leq t \leq T}\|z(t, \cdot)\|_{H}^{p}}, p>2$. We will consider on $\mathfrak{B}_{p, T}$ a closed subset $M$ of such elements $z$ from $\mathfrak{B}_{p, T}$ that $z(t, \cdot)=\phi(t, \cdot)$,

$-r \leq t \leq 0$, and define on $M$ the operator $\Psi$, acting as follows

$$
\begin{aligned}
\Psi u(t, x) & =\int_{\mathbb{R}^{d}} \mathcal{K}(t, x-\xi)\left(\phi(0, \xi)+\int_{\mathbb{R}^{d}} b(0, \xi, \phi(-r, \zeta), \zeta) d \zeta\right) d \xi \\
& -\int_{\mathbb{R}^{d}} b(t, x, u(\alpha(t), \xi), \xi) d \xi \\
& -\int_{0}^{t}\left(\Delta_{x} \int_{\mathbb{R}^{d}} \mathcal{K}(t-s, x-\xi)\left(\int_{\mathbb{R}^{d}} b(s, \xi, u(\alpha(s), \zeta), \zeta) d \zeta\right) d \xi\right) d s \\
& +\int_{0}^{t} \int_{\mathbb{R}^{d}} \mathcal{K}(t-s, x-\xi) f(s, u(\alpha(s), \xi), \xi) d \xi d s \\
& +\int_{0}^{t} \sum_{n=1}^{\infty} \sqrt{\lambda_{n}}\left(\int_{\mathbb{R}^{d}} \mathcal{K}(t-s, x-\xi) \sigma(s, u(\alpha(s), \xi), \xi) e_{n}(\xi) d \xi\right) d \beta_{n}(s) \\
& =\sum_{j=0}^{4} I_{j}(t), 0 \leq t \leq T, x \in \mathbb{R}^{d},
\end{aligned}
$$

and $\Psi u(t, \cdot)=\phi(t, \cdot),-r \leq t \leq T, r>0$. Firstly let show that $\Psi$ takes each $u \in M$ to $\Psi u \in M$. In order to do it, we need to estimate the norms of each of five components in (29).

For $\left\|I_{0}(s)\right\|_{\mathfrak{B}_{p, t}}^{p}=\mathbf{E} \sup _{0 \leq s \leq t}\left\|I_{0}(s)\right\|_{H}^{p}, 0 \leq t \leq T$, we get, using (5),

$$
\left\|I_{0}(s)\right\|_{\mathfrak{B}_{p, t}}^{p} \leq 2^{p-1} \mathbf{E}\|\phi(0, \cdot)\|_{H}^{p}+
$$




$$
+2^{p-1} \mathbf{E}\left\|\int_{\mathbb{R}^{d}}|b(0, \cdot, \phi(-r, \zeta), \zeta)| d \zeta\right\|_{H}^{p}
$$

For the second term in (30) we obtain, applying the conditions from assumptions (6), (5) and (3),

$$
\begin{aligned}
& \mathbf{E}\left\|\int_{\mathbb{R}^{d}}|b(0, \cdot, \phi(-r, \zeta), \zeta)-b(0, \cdot, 0, \zeta)+b(0, \cdot, 0, \zeta)| d \zeta\right\|_{H}^{p} \\
& \quad \leq \mathbf{E}\left\|\int_{\mathbb{R}^{d}}(l(\cdot, \zeta)|\phi(-r, \zeta)|+|b(0, \cdot, 0, \zeta)|) d \zeta\right\|_{H}^{p} \\
& \quad \leq 2^{p-1}\left(\mathbf{E}\left(\iint_{\mathbb{R}^{d}}\left(\int_{\mathbb{R}^{d}} l(x, \zeta)|\phi(-r, \zeta)| d \zeta\right)^{2} d x\right)^{\frac{p}{2}}\right. \\
& \left.\quad+\left(\int_{\mathbb{R}^{d}}\left(\int_{\mathbb{R}^{d}} b_{1}(x, \zeta) d \zeta\right)^{2} d x\right)^{\frac{p}{2}}\right) \\
& \quad \leq 2^{p-1}\left(\int_{\mathbb{R}^{d}} \int_{\mathbb{R}^{d}} l^{2}(x, \zeta) d \zeta d x\right)^{\frac{p}{2}} \mathbf{E}\|\phi(-r, \cdot)\|_{H}^{p} \\
& \left.\quad+\left(\int_{\mathbb{R}^{d}}\left(\int_{\mathbb{R}^{d}} b_{1}(x, \zeta) d \zeta\right)^{2} d x\right)^{\frac{p}{2}}\right)<\infty .
\end{aligned}
$$

For $\left\|I_{1}(s)\right\|_{\mathfrak{B}_{p, t}}^{p}$, as above in (31), we have

$$
\begin{aligned}
\left\|I_{1}(s)\right\|_{\mathfrak{B}_{p, t}}^{p} & =\mathbf{E} \sup _{0 \leq s \leq t}\left\|\int_{\mathbb{R}^{d}} b(s, \cdot, u(\alpha(s), \xi), \xi) d \xi\right\|_{H}^{p} \\
& \leq 2^{p-1}\left(\left(\int_{\mathbb{R}^{d}} \int_{\mathbb{R}^{d}} l^{2}(x, \zeta) d \zeta d x\right)^{\frac{p}{2}} \mathbf{E} \sup _{0 \leq s \leq t}\|u(\alpha(s), \cdot)\|_{H}^{p}\right. \\
& \left.+\left(\int_{\mathbb{R}^{d}}\left(\int_{\mathbb{R}^{d}} b_{1}(x, \zeta) d \zeta\right)^{2} d x\right)^{\frac{p}{2}}\right)<\infty,
\end{aligned}
$$

due to the conditions from assumptions (6), (5) and (1).

Now let estimate $\left\|I_{2}(s)\right\|_{\mathfrak{B}_{p, t}}^{p}$, taking into account the Cauchy-Schwartz inequality and the Fubini theorem,

$\left\|I_{2}(s)\right\|_{\mathfrak{B}_{p, t}}^{p}$

$$
=\mathbf{E} \sup _{0 \leq s \leq t}\left\|\int_{0}^{s}\left(\Delta_{x} \int_{\mathbb{R}^{d}} \mathcal{K}(s-\tau, x-\xi)\left(\int_{\mathbb{R}^{d}} b(\tau, \xi, u(\alpha(\tau), \zeta), \zeta) d \zeta\right) d \xi\right) d \tau\right\|_{H}^{p}
$$




$$
\begin{aligned}
& =\mathbf{E} \sup _{0 \leq s \leq t}\left(\int _ { \mathbb { R } ^ { d } } \left(\int _ { 0 } ^ { s } \left(\Delta_{x} \int_{\mathbb{R}^{d}} \mathcal{K}(s-\tau, x-\xi)\right.\right.\right. \\
& \left.\left.\left.\times\left(\int_{\mathbb{R}^{d}} b(\tau, \xi, u(\alpha(\tau), \zeta), \zeta) d \zeta\right) d \xi\right) d \tau\right)^{2} d x\right)^{\frac{p}{2}} \\
& \leq t^{\frac{p}{2}} \mathbf{E} \sup _{0 \leq s \leq t}\left(\int _ { \mathbb { R } ^ { d } } \int _ { 0 } ^ { s } \left(\Delta_{x} \int_{\mathbb{R}^{d}} \mathcal{K}(s-\tau, x-\xi)\right.\right. \\
& \left.\left.\times\left(\int_{\mathbb{R}^{d}} b(\tau, \xi, u(\alpha(\tau), \zeta), \zeta) d \zeta\right) d \xi\right)^{2} d \tau d x\right)^{\frac{p}{2}} \\
& =t^{\frac{p}{2}} \mathbf{E} \sup _{0 \leq s \leq t}\left(\int _ { 0 } ^ { s } \int _ { \mathbb { R } ^ { d } } \left(\Delta_{x} \int_{\mathbb{R}^{d}} \mathcal{K}(s-\tau, x-\xi)\right.\right. \\
& \left.\left.\times\left(\int_{\mathbb{R}^{d}} b(\tau, \xi, u(\alpha(\tau), \zeta), \zeta) d \zeta\right) d \xi\right)^{2} d x d \tau\right)^{\frac{p}{2}} \\
& \leq t^{\frac{p}{2}} \mathbf{E}\left(\int_{0}^{t} \sup _{0 \leq s \leq t} \| \Delta_{x} \int_{\mathbb{R}^{d}} \mathcal{K}(s-\tau, x-\xi)\right. \\
& \left.\times\left(\int_{\mathbb{R}^{d}} b(\tau, \xi, u(\alpha(\tau), \zeta), \zeta) d \zeta\right) d \xi \|_{H}^{2} d \tau\right)^{\frac{p}{2}} \\
& \leq t^{p-1} \mathbf{E} \int_{0}^{t}\left(\sup _{0 \leq s \leq t} \| \Delta_{x} \int_{\mathbb{R}^{d}} \mathcal{K}(s-\tau, x-\xi)\right. \\
& \left.\times\left(\int_{\mathbb{R}^{d}} b(\tau, \xi, u(\alpha(\tau), \zeta), \zeta) d \zeta\right) d \xi \|_{H}^{2}\right)^{\frac{p}{2}} d \tau .
\end{aligned}
$$

In order to estimate (33), we rely on proposition 6 with

$$
\begin{gathered}
u(s, \tau, x)=\int_{\mathbb{R}^{d}} \mathcal{K}(s-\tau, x-\xi)\left(\int_{\mathbb{R}^{d}} b(\tau, \xi, u(\alpha(\tau), \zeta), \zeta) d \zeta\right) d \xi, \\
g(\tau, x, \omega)=\int_{\mathbb{R}^{d}} b(\tau, x, u(\alpha(\tau), \zeta), \zeta) d \zeta,
\end{gathered}
$$

where $u(s, \tau, x)$ is the solution to $(4)-\left(4^{*}\right)$ with the initial datum in $\tau$. We now need to prove that for any $0 \leq \tau \leq t$

1) $g(\tau, \cdot, \omega) \in L_{1}\left(\mathbb{R}^{d}\right) \cap H$ with probability one;

2) $D_{x}^{2} g(\tau, x, \omega) \in H$. 
1. Due to $(16),(17),(15),(13)$ and belonging $u$ to $M$, we get

$$
\begin{aligned}
& \mathbf{E} \int_{\mathbb{R}^{d}}|g(\tau, x, \omega)| d x \\
& \leq \mathbf{E} \int_{\mathbb{R}^{d}} \int_{\mathbb{R}^{d}}|b(\tau, x, u(\alpha(\tau), \zeta), \zeta)-b(\tau, x, 0, \zeta)+b(\tau, x, 0, \zeta)| d \zeta d x \\
& \leq \mathbf{E} \int_{\mathbb{R}^{d}} \int_{\mathbb{R}^{d}} l(x, \zeta)|u(\alpha(\tau), \zeta)| d \zeta d x+\int_{\mathbb{R}^{d}} \int_{\mathbb{R}^{d}}|b(\tau, x, 0, \zeta)| d \zeta d x \\
& \leq\left(\int_{\mathbb{R}^{d}} \sqrt{\left.\int_{\mathbb{R}^{d}} l^{2}(x, \zeta) d \zeta d x\right) \mathbf{E}\|u(\alpha(\tau), \cdot)\|_{H}}\right. \\
& \quad+\int_{\mathbb{R}^{d}} \int_{\mathbb{R}^{d}} b_{1}(x, \zeta) d \zeta d x<\infty .
\end{aligned}
$$

Hence, $g(\tau, \cdot, \omega)$ belongs to $L_{1}\left(\mathbb{R}^{d}\right)$ for any $0 \leq \tau \leq t$.

Next, taking into account (16), (18), (15), (14) and belonging $u$ to $M$, we conclude

$$
\begin{aligned}
& \mathbf{E} \int_{\mathbb{R}^{d}}|g(\tau, x, \omega)|^{2} d x \leq \mathbf{E} \int_{\mathbb{R}^{d}}\left(\int_{\mathbb{R}^{d}}|b(\tau, x, u(\alpha(\tau), \zeta), \zeta)| d \zeta\right)^{2} d x \\
& \quad \leq 2 \mathbf{E} \int_{\mathbb{R}^{d}}\left(\int_{\mathbb{R}^{d}} l(x, \zeta)|u(\alpha(\tau), \zeta)| d \zeta\right)^{2} d x+2 \iint_{\mathbb{R}^{d}}\left(\int_{\mathbb{R}^{d}}|b(\tau, x, 0, \zeta)| d \zeta\right)^{2} d x \\
& \quad \leq 2\left(\int_{\mathbb{R}^{d}} \int_{\mathbb{R}^{d}} l^{2}(x, \zeta) d \zeta d x\right) \mathbf{E}\|u(\alpha(\tau), \cdot)\|_{H}^{2} \\
& \quad+2 \int_{\mathbb{R}^{d}}\left(\int_{\mathbb{R}^{d}} b_{1}(x, \zeta) d \zeta\right)^{2} d x<\infty .
\end{aligned}
$$

Consequently, condition 1) fulfills.

2. In order to prove item 2), we will use Lebesgue's differentiation theorem on differentiation with respect to a parameter. Let $x_{0}$ be an arbitrary fixed point from $\mathbb{R}^{d}, B_{\delta}\left(x_{0}\right)$ - its vicinity from assumption (7). This assumption implies that the integrand from (34) is differentiable with respect to $x$. From (19) and (23) we have

$$
\begin{aligned}
\left|\nabla_{x} b(\tau, x, u(\alpha(\tau), \zeta), \zeta)\right| & \leq \psi(\tau, x, \zeta)(1+|u(\alpha(\tau), \zeta)|) \\
& \leq\left(\left|\psi(\tau, x, \zeta)-\psi\left(\tau, x_{0}, \zeta\right)\right|+\psi\left(\tau, x_{0}, \zeta\right)\right) \\
& \times(1+|u(\alpha(\tau), \zeta)|) \\
& \leq\left(\delta \varphi\left(\tau, \zeta, x_{0}, \delta\right)+\psi\left(\tau, x_{0}, \zeta\right)\right) \\
& \times(1+|u(\alpha(\tau), \zeta)|)
\end{aligned}
$$


We claim that the expression on the right-hand of (37) is integrable. Indeed, by virtue of belonging $u$ to $M$ and estimates (22), (20), (21), we obtain

$$
\begin{aligned}
& \mathbf{E} \int_{\mathbb{R}^{d}}\left(\varphi\left(\tau, \zeta, x_{0}, \delta\right)+\psi\left(\tau, x_{0}, \zeta\right)\right)(1+|u(\alpha(\tau), \zeta)|) d \zeta \\
& \quad \leq \int_{\mathbb{R}^{d}} \varphi\left(\tau, \zeta, x_{0}, \delta\right) d \zeta+\int_{\mathbb{R}^{d}} \psi\left(\tau, x_{0}, \zeta\right) d \zeta \\
& \quad+\left(\sqrt{\int_{\mathbb{R}^{d}} \varphi^{2}\left(\tau, \zeta, x_{0}, \delta\right) d \zeta}+\sqrt{\int_{\mathbb{R}^{d}} \psi^{2}\left(\tau, x_{0}, \zeta\right) d \zeta}\right) \\
& \quad \times \sqrt{\mathbf{E}\|u(\alpha(\tau), \cdot)\|_{H}^{2}}<\infty .
\end{aligned}
$$

Hence, the function $\left|\nabla_{x} b(\tau, x, u(\alpha(\tau), \zeta), \zeta)\right|$ is bounded above by the integrable function $\left(\varphi\left(\tau, \zeta, x_{0}, \delta\right)+\psi\left(\tau, x_{0}, \zeta\right)\right) \times$ $\times(1+|u(\alpha(\tau), \zeta)|)$. Hence, $g(\tau, x, \omega)$ is differentiable with respect to $x$.

Now it remains to check that for any $0 \leq \tau \leq T \nabla g(\tau, \cdot, \omega)$ belongs to $H$ with probability one. Indeed, taking into account estimates (19), (20), (21) and belonging $u$ to $M$, we obtain

$$
\begin{aligned}
& \mathbf{E} \int_{\mathbb{R}^{d}}\left|\nabla_{x} \int_{\mathbb{R}^{d}} b(\tau, x, u(\alpha(\tau), \zeta), \zeta) d \zeta\right|^{2} d x \\
& \leq \mathbf{E} \int_{\mathbb{R}^{d}}\left(\int_{\mathbb{R}^{d}} \psi(\tau, x, \zeta)(1+|u(\alpha(\tau), \zeta)|) d \zeta\right)^{2} d x \\
& \leq 2 \int_{\mathbb{R}^{d}}\left(\int_{\mathbb{R}^{d}} \psi(\tau, x, \zeta) d \zeta\right)^{2} d x+2\left(\int_{\mathbb{R}^{d}} \int_{\mathbb{R}^{d}} \psi^{2}(\tau, x, \zeta) d \zeta d x\right) \\
& \quad \times \mathbf{E}\|u(\alpha(\tau), \cdot)\|_{H}^{2} \leq \text { const. }
\end{aligned}
$$

Existence of $D_{x}^{2} g(\tau, x, \omega)$ and validity of the inequality

$$
\mathbf{E} \int_{\mathbb{R}^{d}}\left\|D_{x}^{2} \int_{\mathbb{R}^{d}} b(\tau, x, u(\alpha(\tau), \zeta), \zeta) d \zeta\right\|_{d}^{2} d x \leq \text { const }
$$

is proved as above.

Thus, taking into account (9) and conditions 1), 2), we conclude that the righthand of (33) is estimated by the expression

$$
C_{T}^{\frac{p}{2}} t^{p-1} \int_{0}^{t} \mathbf{E}\left(\int_{\mathbb{R}^{d}}\left\|D_{x}^{2} \int_{\mathbb{R}^{d}} b(\tau, x, u(\alpha(\tau), \zeta), \zeta) d \zeta\right\|_{d}^{2} d x\right)^{\frac{p}{2}} d \tau .
$$


Similarly to (38), taking into account that $u \in M$ and estimates (19), (20), (21), we can easily obtain the following estimate

$$
\begin{aligned}
& \mathbf{E}\left(\int_{\mathbb{R}^{d}}\left\|D_{x}^{2} \int_{\mathbb{R}^{d}} b(\tau, x, u(\alpha(\tau), \zeta), \zeta) d \zeta\right\|_{d}^{2} d x\right)^{\frac{p}{2}} \\
& \quad \leq \mathbf{E}\left(\int_{\mathbb{R}^{d}}\left(\int_{\mathbb{R}^{d}} \psi(\tau, x, \zeta)(1+|u(\alpha(\tau), \zeta)|) d \zeta\right)^{2} d x\right)^{\frac{p}{2}} \\
& \quad \leq 2^{p-1} \mathbf{E}\left(\left(\int_{\mathbb{R}^{d}}\left(\int_{\mathbb{R}^{d}} \psi(\tau, x, \zeta) d \zeta\right)^{2} d x\right)^{\frac{p}{2}}\right. \\
& \left.\quad+\left(\int_{\mathbb{R}^{d}} \int_{\mathbb{R}^{d}} \psi^{2}(\tau, x, \zeta) d \zeta d x\right)^{\frac{p}{2}}\|u(\alpha(\tau), \cdot)\|_{H}^{p}\right) \leq \text { const. }
\end{aligned}
$$

Substituting (40) into (39), we have

$$
\left\|I_{2}(s)\right\|_{\mathfrak{B}_{p, t}}^{p}<\infty .
$$

Next let estimate $\left\|I_{3}(s)\right\|_{\mathfrak{B}_{p, t}}^{p}$. Taking into account (5), (11), (10) and belonging $u$ to $M$, we compute

$$
\begin{aligned}
\left\|I_{3}(s)\right\|_{\mathfrak{B}_{p, t}}^{p} & =\mathbf{E} \sup _{0 \leq s \leq t}\left(\int_{\mathbb{R}^{d}}\left(\int_{0}^{s} \int_{\mathbb{R}^{d}} \mathcal{K}(s-\tau, x-\xi) f(\tau, u(\alpha(\tau), \xi), \xi) d \xi d \tau\right)^{2} d x\right)^{\frac{p}{2}} \\
& \leq t^{\frac{p}{2}} \mathbf{E} \sup _{0 \leq s \leq t}\left(\int_{0}^{s} \int_{\mathbb{R}^{d}}\left(\int_{\mathbb{R}^{d}} \mathcal{K}(s-\tau, x-\xi) f(\tau, u(\alpha(\tau), \xi), \xi) d \xi\right)^{2} d x d \tau\right)^{\frac{p}{2}} \\
& \leq t^{\frac{p}{2}} \mathbf{E}\left(\int_{0}^{t}\|f(\tau, u(\alpha(\tau), \cdot), \cdot)\|_{H}^{2} d \tau\right)^{\frac{p}{2}} \\
& \leq t^{p-1} \mathbf{E} \int_{0}^{t}\|f(\tau, u(\alpha(\tau), \cdot), \cdot)\|_{H}^{p} d \tau \\
& \leq 2^{p-1} t^{p-1}\left(\int_{0}^{t}\left(\int_{\mathbb{R}^{d}} \chi^{2}(\tau, x) d x\right)^{\frac{p}{2}} d \tau+L^{p} \int_{0}^{t} \mathbf{E}\|u(\alpha(\tau), \cdot)\|_{H}^{p} d \tau\right) \\
& <\infty .
\end{aligned}
$$

It remains to estimate the last term, $\left\|I_{4}(s)\right\|_{\mathfrak{B}_{p, t}}^{p}$. From [4, proposition 7.3] we have

$$
\mathbf{E} \sup _{0 \leq s \leq t}\left\|\int_{0}^{s} S(s-\tau) \sigma(\tau, u(\alpha(\tau), \cdot), \cdot) d W(\tau)\right\|_{H}^{p}
$$




$$
\leq D(T) \int_{0}^{t} \mathbf{E}\|\sigma(s, u(\alpha(s), \cdot), \cdot)\|_{L_{2}^{0}}^{p} d s
$$

for some $D(T)>0$. We obtain the consequence of the following computations for the Hilbert-Schmidt norm of the integrand on the right-hand of (42)

$$
\begin{aligned}
& \mathbf{E}\|\sigma(s, u(\alpha(s), \cdot), \cdot)\|_{L_{2}^{0}}^{p} \\
& =\mathbf{E}\left(\sum_{n=1}^{\infty}\left\|\sigma(s, u(\alpha(s), \cdot), \cdot) Q^{\frac{1}{2}} e_{n}(\cdot)\right\|_{H}^{2}\right)^{\frac{p}{2}} \\
& =\mathbf{E}\left(\sum_{n=1}^{\infty} \lambda_{n}\left\|\sigma(s, u(\alpha(s), \cdot), \cdot) e_{n}(\cdot)\right\|_{H}^{2}\right)^{\frac{p}{2}} \\
& =\mathbf{E}\left(\sum_{n=1}^{\infty} \lambda_{n} \int_{\mathbb{R}^{d}} \sigma^{2}(s, u(\alpha(s), x), x) e_{n}^{2}(x) d x\right)^{\frac{p}{2}} \\
& \leq L^{p} \mathbf{E}\left(\sum_{n=1}^{\infty} \lambda_{n}\left(\int_{\mathbb{R}^{d}} e_{n}^{2}(x) d x+\int_{\mathbb{R}^{d}} u^{2}(\alpha(s), x) d x\right)\right)^{\frac{p}{2}} \\
& \leq 2^{\frac{p}{2}-1} \lambda^{\frac{p}{2}} L^{p}\left(1+\mathbf{E}\|u(\alpha(s), \cdot)\|_{H}^{p}\right),
\end{aligned}
$$

thus the right-hand of (42) is bounded.

Now let prove that trajectories of the process $\Psi u$ from (29) are continuous.

The first term, $I_{0}$, is continuous due to proposition 1 and because the expression $\phi(0, \cdot)+\int_{\mathbb{R}^{d}} b(0, \cdot, \phi(-r, \zeta), \zeta) d \zeta$ belongs to $H$ with probability one.

Now we claim that the second term, $I_{1}$, is continuous. Indeed, let $0 \leq t_{0} \leq T$ be fixed. We have

$$
\begin{aligned}
& \int_{\mathbb{R}^{d}}\left(\int_{\mathbb{R}^{d}}\left(b(t, x, u(\alpha(t), \xi), \xi)-b\left(t_{0}, x, u\left(\alpha\left(t_{0}\right), \xi\right), \xi\right)\right) d \xi\right)^{2} d x \\
& \quad \leq 2 \iint_{\mathbb{R}^{d}}\left(\int_{\mathbb{R}^{d}}\left|b(t, x, u(\alpha(t), \xi), \xi)-b\left(t, x, u\left(\alpha\left(t_{0}\right), \xi\right), \xi\right)\right| d \xi\right)^{2} d x \\
& \quad+2 \int_{\mathbb{R}^{d}}\left(\int_{\mathbb{R}^{d}}\left|b\left(t, x, u\left(\alpha\left(t_{0}\right), \xi\right), \xi\right)-b\left(t_{0}, x, u\left(\alpha\left(t_{0}\right), \xi\right), \xi\right)\right| d \xi\right)^{2} d x \\
& \quad=J_{1}(t)+J_{2}(t) .
\end{aligned}
$$

Let estimate each of two terms in (44) separately. Taking into account (16), the Cauchy-Swartz inequality, (18) and belonging $u$ to $M$, we obtain with probability one

$$
J_{1}(t) \leq 2 \int_{\mathbb{R}^{d}}\left(\int_{\mathbb{R}^{d}} l(x, \xi)\left|u(\alpha(t), \xi)-u\left(\alpha\left(t_{0}\right), \xi\right)\right| d \xi\right)^{2} d x
$$




$$
\begin{aligned}
& \leq 2\left(\int_{\mathbb{R}^{d}} \int_{\mathbb{R}^{d}} l^{2}(x, \xi) d \xi d x\right)\left\|u(\alpha(t), \cdot)-u\left(\alpha\left(t_{0}\right), \cdot\right)\right\|_{H}^{2} \\
& \underset{t \rightarrow t_{0}}{ } 0 .
\end{aligned}
$$

Now let show that $J_{2}(t)$ is tending to zero as $t \rightarrow t_{0}$ with probability one. In order to do it, we need to substantiate a capability of limit passage in this integral. Firstly, we claim that for all $x \in \mathbb{R}^{d}$ the expression

$$
\varphi(t, x)=\int_{\mathbb{R}^{d}}\left|b\left(t, x, u\left(\alpha\left(t_{0}\right), \xi\right), \xi\right)-b\left(t_{0}, x, u\left(\alpha\left(t_{0}\right), \xi\right), \xi\right)\right| d \xi
$$

is tending to zero as $t \rightarrow t_{0}$ with probability one. Indeed, since $b$ is continuous in the first argument, the integrand on the right-hand of (46) is tending to zero. Then, taking into account (16) and (15), we have

$$
\left|b\left(t, x, u\left(\alpha\left(t_{0}\right), \xi\right), \xi\right)\right| \leq l(x, \xi)\left|u\left(\alpha\left(t_{0}\right), \xi\right)\right|+b_{1}(x, \xi) .
$$

Condition (14) guarantees that $\int_{\mathbb{R}^{d}} b_{1}(x, \xi) d \xi<\infty$ for any fixed $x \in \mathbb{R}^{d}$. Next, taking into account the Cauchy-Schwartz inequality, condition (17) and belonging $u$ to $M$, we calculate

$$
\int_{\mathbb{R}^{d}} l(x, \xi)\left|u\left(\alpha\left(t_{0}\right), \xi\right)\right| d \xi \leq \sqrt{\int_{\mathbb{R}^{d}} l^{2}(x, \xi) d \xi} \cdot\left\|u\left(\alpha\left(t_{0}\right), \cdot\right)\right\|_{H}<\infty .
$$

Therefore the right-hand of (47) is an integrable majorant function for $b$. It now follows that $\varphi(t, x) \underset{t \rightarrow t_{0}}{\rightarrow} 0$ for all $x \in \mathbb{R}^{d}$ and almost all $\omega \in \Omega$.

Let prove that the expression $2\left(\int_{\mathbb{R}^{d}}\left(b_{1}(\cdot, \xi)+l(\cdot, \xi)\left|u\left(\alpha\left(t_{0}\right), \xi\right)\right|\right) d \xi\right)^{2}$ is an integrable majorant for the integrand in $J_{2}(t)$. Indeed, using (14), (18) and taking into account belonging $u$ to $M$, we get

$$
\begin{aligned}
& \int_{\mathbb{R}^{d}}\left(\int_{\mathbb{R}^{d}}\left(b_{1}(x, \xi)+l(x, \xi)\left|u\left(\alpha\left(t_{0}\right), \xi\right)\right|\right) d \xi\right)^{2} d x \\
& \quad \leq 2 \int_{\mathbb{R}^{d}}\left(\int_{\mathbb{R}^{d}} b_{1}(x, \xi)\right)^{2} d x+2 \iint_{\mathbb{R}^{d}}\left(\int_{\mathbb{R}^{d}} l(x, \xi)\left|u\left(\alpha\left(t_{0}\right), \xi\right)\right| d \xi\right)^{2} d x \\
& \quad \leq 2 \int_{\mathbb{R}^{d}}\left(\int_{\mathbb{R}^{d}} b_{1}(x, \xi)\right)^{2} d x+2\left(\int_{\mathbb{R}^{d}} \int_{\mathbb{R}^{d}} l^{2}(x, \xi) d \xi d x\right)\left\|u\left(\alpha\left(t_{0}\right), \cdot\right)\right\|_{H}^{2} \\
& \quad<\infty
\end{aligned}
$$

with probability one. 
Consequently, $J_{1}(t)+J_{2}(t) \underset{t \rightarrow t_{0}}{\rightarrow} 0$ and $I_{1}(t)$ is continuous in $t$.

It is obvious that $I_{2}$ and $I_{3}$ are continuous. Therefore it only remains to prove that $I_{4}$ is continuous. In order to do it, we will use the factorization method from [3, theorem 5.2.5]. First we need to show that $\mathbf{E}\|S(s-\tau) \sigma(\tau, u(\alpha(\tau), \cdot), \cdot) d W(\tau)\|_{L_{2}^{0}}^{2}$ is bounded. Similarly to (43), we compute

$$
\begin{aligned}
& \mathbf{E}\|S(s-\tau) \sigma(\tau, u(\alpha(\tau), \cdot), \cdot)\|_{L_{2}^{0}}^{2} \\
& =\mathbf{E} \sum_{n=1}^{\infty} \lambda_{n}\left\|S(s-\tau) \sigma(\tau, u(\alpha(\tau), \cdot), \cdot) e_{n}(\cdot)\right\|_{H}^{2} \\
& \leq \mathbf{E} \sum_{n=1}^{\infty} \lambda_{n} \int_{\mathbb{R}^{d}} \sigma^{2}(\tau, u(\alpha(\tau), x), x) e_{n}^{2}(x) d x \\
& \leq L^{2} \mathbf{E}\left(\sum_{n=1}^{\infty} \lambda_{n}\left(\int_{\mathbb{R}^{d}} e_{n}^{2}(x) d x+\int_{\mathbb{R}^{d}} u^{2}(\alpha(\tau), x) d x\right)\right) \\
& \leq \lambda L^{2}\left(1+\mathbf{E}\|u(\alpha(\tau), \cdot)\|_{H}^{2}\right)=B<\infty .
\end{aligned}
$$

Then, from the mentioned above theorem, we have

$$
\begin{aligned}
& \int_{0}^{s} S(s-\tau) \sigma(\tau, u(\alpha(\tau), \cdot), \cdot) d W(\tau) \\
& =\frac{\sin \pi \gamma}{\pi} \int_{0}^{s}(s-\tau)^{\gamma-1} S(s-\tau) U_{\gamma}(\tau) d W(\tau),
\end{aligned}
$$

where

$$
\begin{aligned}
U_{\gamma}(s) & =\int_{0}^{s}(s-\tau)^{-\gamma} S(s-\tau) \sigma(\tau, u(\alpha(\tau), \cdot), \cdot) d W(\tau), \\
\frac{1}{p} & <\gamma<\frac{1}{2} .
\end{aligned}
$$

According to proposition 3 and the mentioned above theorem, in order to prove that $I_{4}$ is continuous, it is enough to show that process (50) has $p$-integrable trajectories. Similarly to (42), we have

$$
\begin{gathered}
\mathbf{E} \sup _{0 \leq s \leq t}\left\|\int_{0}^{s}(s-\tau)^{-\gamma} S(s-\tau) \sigma(\tau, u(\alpha(\tau), \cdot), \cdot) d W(\tau)\right\|_{H}^{p} \\
\quad \leq C_{p} \mathbf{E}\left(\int_{0}^{t}(t-s)^{-2 \gamma}\|S(t-s) \sigma(s, u(\alpha(s), \cdot), \cdot)\|_{L_{2}^{0}}^{2} d s\right)^{\frac{p}{2}}
\end{gathered}
$$




$$
\leq B^{\frac{p}{2}} C_{p}\left(\int_{0}^{t}(t-s)^{-2 \gamma} d s\right)^{\frac{p}{2}}
$$

where $C_{p}$ is a positive number and $B$ is defined from (49). Then we get

$$
\mathbf{E} \int_{0}^{t}\left\|U_{\gamma}(\tau)\right\|_{\mathfrak{B}_{p, s}}^{p} d s \leq B^{\frac{p}{2}} C_{p} \int_{0}^{t}\left(\int_{0}^{s}(s-\tau)^{-2 \gamma} d \tau\right)^{\frac{p}{2}} d s<\infty,
$$

hence $I_{4}$ is continuous with respect to the norm of $H$.

Now, taking into account the proven fact of continuity and combining estimates (30), (31), (32), (33), (39), (40), (41), (42) and (43), we conclude that the operator $\Psi$, defined by (29), transforms $M$ into itself.

Now let prove that this operator is contractive. Let $u$ and $v$ be two elements from $M$. From (29) we obtain

$$
\begin{aligned}
\| \Psi u & -\Psi v \|_{\mathfrak{B}_{p, t}}^{p} \\
& \leq 4^{p-1}\left\|I_{1}(s)(u)-I_{1}(s)(v)\right\|_{\mathfrak{B}_{p, t}}^{p}+4^{p-1}\left\|I_{2}(s)(u)-I_{2}(s)(v)\right\|_{\mathfrak{B}_{p, t}}^{p} \\
& +4^{p-1}\left\|I_{3}(s)(u)-I_{3}(s)(v)\right\|_{\mathfrak{B}_{p, t}}^{p}+4^{p-1}\left\|I_{4}(s)(u)-I_{4}(s)(v)\right\|_{\mathfrak{B}_{p, t}}^{p} .
\end{aligned}
$$

In view of the obtained for $\left\|I_{1}(s)\right\|_{\mathfrak{B}_{p, t}}^{p}$ estimate, we evidently get

$$
\begin{aligned}
\| I_{1}(s)(u) & -I_{1}(s)(v) \|_{\mathfrak{B}_{p, t}}^{p} \leq\left(\int_{\mathbb{R}^{d}} \int_{\mathbb{R}^{d}} l^{2}(x, \xi) d \xi d x\right)^{\frac{p}{2}} \\
\times\|u-v\|_{\mathfrak{B}_{p, t}}^{p} . &
\end{aligned}
$$

Similarly to estimating $I_{2}$ from (29), we obtain

$$
\begin{aligned}
\| I_{2}(s)(u) & -I_{2}(s)(v) \|_{\mathfrak{B}_{p, t}}^{p} \\
& \leq C_{T}^{\frac{p}{2}} t^{p} \sup _{0 \leq \tau \leq t}\left(\int_{\mathbb{R}^{d}} \int_{\mathbb{R}^{d}} \psi^{2}(\tau, x, \zeta) d \zeta d x\right)^{\frac{p}{2}}\|u-v\|_{\mathfrak{B}_{p, t}}^{p} .
\end{aligned}
$$

In a similar way to (41), (42) and (43), we have

$$
\begin{aligned}
& \left\|I_{3}(s)(u)-I_{3}(s)(v)\right\|_{\mathfrak{B}_{p, t}}^{p} \leq L^{p} t^{p}\|u-v\|_{\mathfrak{B}_{p, t}}^{p}, \\
& \left\|I_{4}(s)(u)-I_{4}(s)(v)\right\|_{\mathfrak{B}_{p, t}}^{p} \leq D(T) \lambda^{\frac{p}{2}} L^{p} t\|u-v\|_{\mathfrak{B}_{p, t}}^{p} .
\end{aligned}
$$

Thus, from (51) - (54) we deduce

$$
\begin{aligned}
\| \Psi u & -\Psi v \|_{\mathfrak{B}_{p, t}^{p}}^{p} \\
& \leq 4^{p-1}\left(\left(\int_{\mathbb{R}^{d}} \int_{\mathbb{R}^{d}} l^{2}(x, \xi) d \xi d x\right)^{\frac{p}{2}}+C_{T}^{\frac{p}{2}} t^{p} \sup _{0 \leq \tau \leq t}\left(\int_{\mathbb{R}^{d}} \int_{\mathbb{R}^{d}} \psi^{2}(\tau, x, \zeta) d \zeta d x\right)^{\frac{p}{2}}\right.
\end{aligned}
$$




$$
\left.+L^{p} t^{p}+D(T) \lambda^{\frac{p}{2}} L^{p} t\right)\|u-v\|_{\mathfrak{B}_{p, t}}^{p}=\gamma(t)\|u-v\|_{\mathfrak{B}_{p, t}}^{p} .
$$

According to condition (25), the first term in $\gamma$ from (55) is less than one. Therefore, by choosing small $0 \leq t \leq T$, we conclude that $0<\gamma(t)<1$. It means that the operator $\Psi$, defined in a complete Banach space $\mathfrak{B}_{p, t}$, is contractive, and therefore has a unique fixed point - the mild solution of $(1)-\left(1^{*}\right)$ on $[-r, t]$. This procedure can be repeated finitely many steps in order to extend the solution to the entire interval $[-r, T]$. This completes the proof of the theorem.

While analysing the above proof, we conclude that, under the conditions of corollary 11 , this proof is true on any interval $[0, T]$ for arbitrary $T$. Hence, corollary 11 holds.

\subsection{PROOF OF THEOREM 10}

Let $\phi$ and $\phi_{1}$ be arbitrary admissible functions, $u(t, \cdot, \phi)$ and $u\left(t, \cdot, \phi_{1}\right)$ - the solutions of (1), corresponding to these functions. We get

$$
\begin{aligned}
& u(t, x, \phi)-u\left(t, x, \phi_{1}\right) \\
& =\int_{\mathbb{R}^{d}} \mathcal{K}(t, x-\xi)\left(\phi(0, \xi)-\phi_{1}(0, \xi)\right. \\
& \left.+\int_{\mathbb{R}^{d}}\left(b(0, \xi, \phi(-r, \zeta), \zeta)-b\left(0, \xi, \phi_{1}(-r, \zeta), \zeta\right)\right) d \zeta\right) d \xi \\
& +\int_{\mathbb{R}^{d}}\left(b\left(t, x, u\left(\alpha(t), \xi, \phi_{1}\right), \xi\right)-b(t, x, u(\alpha(t), \xi, \phi), \xi)\right) d \xi \\
& +\int_{0}^{t}\left(\Delta_{x} \int_{\mathbb{R}^{d}} \mathcal{K}(t-s, x-\xi)\right. \\
& \left.\left.\times \int_{\mathbb{R}^{d}}\left(b\left(s, \xi, u\left(\alpha(s), \zeta, \phi_{1}\right), \zeta\right)-b(s, \xi, u(\alpha(s), \zeta, \phi), \zeta)\right) d \zeta\right) d \xi\right) d s \\
& +\int_{0}^{t} \int_{\mathbb{R}^{d}} \mathcal{K}(t-s, x-\xi) \\
& \times\left(f(s, u(\alpha(s), \xi, \phi), \xi)-f\left(s, u\left(\alpha(s), \xi, \phi_{1}\right), \xi\right)\right) d \xi d s \\
& +\int_{0}^{t} \sum_{n=1}^{\infty} \sqrt{\lambda_{n}}\left(\int_{\mathbb{R}^{d}} \mathcal{K}(t-s, x-\xi)\right. \\
& \left.\times\left(\sigma(s, u(\alpha(s), \xi, \phi), \xi)-\sigma\left(s, u\left(\alpha(s), \xi, \phi_{1}\right), \xi\right)\right) e_{n}(\xi) d \xi\right) d \beta_{n}(s) \\
& \quad(f)
\end{aligned}
$$




$$
=\sum_{j=0}^{4} J_{j}(t), 0 \leq t \leq T .
$$

Let estimate $\mathbf{E} \sup _{-r \leq s \leq t}\left\|J_{j}(s)\right\|_{H}^{p}, j \in\{0, \ldots, 4\}$, from each of terms in (56).

Similarly to $(30),(31)$, we have for some $C_{1}>0$

$$
\begin{aligned}
& \mathbf{E} \sup _{-r \leq s \leq t}\left\|J_{0}(s)\right\|_{H}^{p} \\
& \leq 2^{p-1} \mathbf{E}\left\|\phi(0, \cdot)-\phi_{1}(0, \cdot)\right\|_{H}^{p} \\
& +2^{p-1}\left(\int_{\mathbb{R}^{d}} \int_{\mathbb{R}^{d}} l^{2}(x, \zeta) d \zeta d x\right)^{\frac{p}{2}} \mathbf{E}\left\|\phi(-r, \cdot)-\phi_{1}(-r, \cdot)\right\|_{H}^{p} \\
& \quad \leq C_{1} \mathbf{E} \sup _{-r \leq s \leq 0}\left\|\phi(s, \cdot)-\phi_{1}(s, \cdot)\right\|_{H}^{p} .
\end{aligned}
$$

Using (51), we deduce

$$
\begin{aligned}
& \mathbf{E} \sup _{-r \leq s \leq t}\left\|J_{1}(s)\right\|_{H}^{p} \leq\left(\int_{\mathbb{R}^{d}} \int_{\mathbb{R}^{d}} l^{2}(x, \zeta) d \zeta d x\right)^{\frac{p}{2}} \\
& \quad \times \mathbf{E} \sup _{-r \leq s \leq t}\left\|u\left(\alpha(s), \cdot, \phi_{1}\right)-u(\alpha(s), \cdot, \phi)\right\|_{H}^{p} \leq\left(\iint_{\mathbb{R}^{d}} l_{\mathbb{R}^{d}}(x, \zeta) d \zeta d x\right)^{\frac{p}{2}} \\
& \quad \times \mathbf{E} \sup _{-r \leq s \leq t}\left\|u\left(s, \cdot, \phi_{1}\right)-u(s, \cdot, \phi)\right\|_{H}^{p} .
\end{aligned}
$$

Now let estimate $\mathbf{E} \sup _{-r \leq s \leq t}\left\|J_{2}(s)\right\|_{H}^{p}$, taking into account estimate of the right-hand on (33) (namely, estimates (39) and (40)). We get

$$
\begin{aligned}
& \mathbf{E} \sup _{-r \leq s \leq t}\left\|J_{2}(s)\right\|_{H}^{p} \\
& \leq C_{T}^{\frac{p}{2}} t^{p-1} \\
& \times \int_{0}^{t} \mathbf{E}\left(\int_{\mathbb{R}^{d}}\left(\int_{\mathbb{R}^{d}}\left\|D_{x}^{2} b\left(\tau, x, u\left(\alpha(\tau), \zeta, \phi_{1}\right), \zeta\right)-D_{x}^{2} b(\tau, x, u(\alpha(\tau), \zeta, \phi), \zeta)\right\|_{d} d \zeta\right)^{2} d x\right)^{\frac{p}{2}} d \tau \\
& \leq C_{T}^{\frac{p}{2}} t^{p-1} \sup _{0 \leq \tau \leq T}\left(\int_{\mathbb{R}^{d}} \int_{\mathbb{R}^{d}} \psi^{2}(\tau, x, \zeta) d \zeta d x\right)^{\frac{p}{2}} \\
& \times \int_{0}^{t} \mathbf{E} \sup _{-r \leq \tau \leq s}\left\|u\left(\alpha(\tau), \cdot, \phi_{1}\right)-u(\alpha(\tau), \cdot, \phi)\right\|_{H}^{p} d s \\
& \leq C_{T}^{\frac{p}{2}} t^{p-1} \sup _{0 \leq \tau \leq T}\left(\int_{\mathbb{R}^{d}} \int_{\mathbb{R}^{d}} \psi^{2}(\tau, x, \zeta) d \zeta d x\right)^{\frac{p}{2}}
\end{aligned}
$$


$\times \int_{0}^{t} \mathbf{E} \sup _{-r \leq \tau \leq s}\left\|u\left(\tau, \cdot, \phi_{1}\right)-u(\tau, \cdot, \phi)\right\|_{H}^{p} d s$.

Using (41) for estimating $\mathbf{E} \sup _{-r \leq s \leq t}\left\|J_{3}(s)\right\|_{H}^{p}$, we can easily obtain the following

$$
\begin{aligned}
\mathbf{E} & \sup _{-r \leq s \leq t}\left\|J_{3}(s)\right\|_{H}^{p} \\
& \leq L^{p} t^{p-1} \int_{0}^{t} \mathbf{E} \sup _{-r \leq \tau \leq s}\left\|u\left(\alpha(\tau), \cdot, \phi_{1}\right)-u(\alpha(\tau), \cdot, \phi)\right\|_{H}^{p} d s \\
& \leq L^{p} t^{p-1} \int_{0}^{t} \mathbf{E} \sup _{-r \leq \tau \leq s}\left\|u\left(\tau, \cdot, \phi_{1}\right)-u(\tau, \cdot, \phi)\right\|_{H}^{p} d s .
\end{aligned}
$$

Similarly, using (42) and (43), for $\mathbf{E} \sup _{-r \leq s \leq t}\left\|J_{4}(s)\right\|_{H}^{p}$ we have the following estimate

$$
\begin{aligned}
& \mathbf{E} \sup _{-r \leq s \leq t}\left\|J_{4}(s)\right\|_{H}^{p} \\
& \leq D(T) L^{p} \lambda^{\frac{p}{2}} \int_{0}^{t} \mathbf{E} \sup _{-r \leq \tau \leq s}\left\|u\left(\alpha(\tau), \cdot, \phi_{1}\right)-u(\alpha(\tau), \cdot, \phi)\right\|_{H}^{p} d s \\
& \leq D(T) L^{p} \lambda^{\frac{p}{2}} \int_{0}^{t} \mathbf{E} \sup _{-r \leq \tau \leq s}\left\|u\left(\tau, \cdot, \phi_{1}\right)-u(\tau, \cdot, \phi)\right\|_{H}^{p} d s .
\end{aligned}
$$

Thus, from (58) - (61) it is now clear that for some $C_{2}>0$

$$
\begin{aligned}
\mathbf{E} & \sup _{-r \leq s \leq t}\left\|u\left(s, \cdot, \phi_{1}\right)-u(\tau, \cdot, \phi)\right\|_{H}^{p} \\
\leq & C_{1} \mathbf{E} \sup _{-r \leq s \leq 0}\left\|\phi_{1}(s, \cdot)-\phi(s, \cdot)\right\|_{H}^{p} \\
+ & \left(\int_{\mathbb{R}^{d}} \int_{\mathbb{R}^{d}} l^{2}(x, \zeta) d \zeta d x\right)^{\frac{p}{2}} \mathbf{E} \sup _{-r \leq s \leq t}\left\|u\left(s, \cdot, \phi_{1}\right)-u(s, \cdot, \phi)\right\|_{H}^{p} \\
+ & C_{2} \int_{0}^{t} \mathbf{E} \sup _{-r \leq \tau \leq s}\left\|u\left(\tau, \cdot, \phi_{1}\right)-u(\tau, \cdot, \phi)\right\|_{H}^{p} d s .
\end{aligned}
$$

In view of (25), we conclude

$$
\begin{aligned}
& \mathbf{E} \sup _{-r \leq s \leq t}\left\|u\left(s, \cdot, \phi_{1}\right)-u(\tau, \cdot, \phi)\right\|_{H}^{p} \\
& \leq C_{3} \mathbf{E} \sup _{-r \leq s \leq 0}\left\|\phi_{1}(s, \cdot)-\phi(s, \cdot)\right\|_{H}^{p} \\
& +C_{4} \int_{0}^{t} \mathbf{E} \sup _{-r \leq \tau \leq s}\left\|u\left(\tau, \cdot, \phi_{1}\right)-u(\tau, \cdot, \phi)\right\|_{H}^{p} d s,
\end{aligned}
$$


where $C_{3}=\frac{C_{1}}{1-\left(\int_{\mathbb{R}^{d}} \int_{\mathbb{R}^{d}} l^{2}(x, \zeta) d \zeta d x\right)^{\frac{p}{2}}}, C_{4}=\frac{C_{2}}{1-\left(\int_{\mathbb{R}^{d}} \int_{\mathbb{R}^{d}} l^{2}(x, \zeta) d \zeta d x\right)^{\frac{p}{2}}}$. Application of Gronwall's inequality to (62) gives (26), thereby concluding the proof.

\subsection{PROOF OF THEOREM 13}

Let $u(t, s, \cdot, \phi)$ be the mild solution of $(1)-\left(1^{*}\right)$ in the terms of section 3, i.e. let $u(s+\theta, s, \cdot, \phi)=\phi(s+\theta, \cdot),-r \leq \theta \leq 0$, and $u(t, s, \cdot, \phi)$ satisfy (24) for $t \geq s$. Here the function $\phi(s+\theta, \cdot, \omega)$ is $\mathcal{F}_{s}$-measurable and satisfies the conditions from assumption (3) for any fixed $s$ such that $0 \leq s \leq t \leq T$. From theorem 9 we conclude that for fixed $s$ and $t u_{t}(s, \cdot, \phi)=u(t+\theta, s, \cdot, \phi)$ as a function of $\theta$ is a random variable from $\mathfrak{C}$.

Let $\varphi \in \mathfrak{C}$ be non-random. Then $u(t, s, \cdot, \varphi)$ is completely defined by the increments $W(\tau)-W(s), \tau \geq s$, therefore it does not depend on $\sigma$-algebra $\mathcal{F}_{s}$ and is $\mathcal{G}_{s}$-measurable. Here $\mathcal{G}_{s}$ is the minimal $\sigma$-algebra, generated by the increments $W(\tau)-W(s), \tau \geq s$. It is easy to see that, due to theorem 9 , proposition 12 is true for a random initial $\mathcal{F}_{s}$-measurable function $\phi$ such that $\mathbf{E} \sup _{-r \leq t \leq T}\|\phi(t, \cdot)\|_{H}^{p}<\infty$, $p>2$. Hence, for any $0 \leq s \leq \tau \leq t \leq T$ we have

$$
u_{t}(s, \cdot, \phi)=u_{t}\left(\tau, \cdot, u_{\tau}(s, \cdot, \phi)\right)
$$

Note that $u_{\tau}(s, \cdot, \phi)$ is $\mathcal{F}_{\tau}$-measurable function and does not depend on $\sigma$-algebra $\mathcal{G}_{\tau}$.

Thus, $u_{t}(s, \cdot, \phi)=\beta\left(u_{\tau}(s, \cdot, \phi), \omega\right)$, where $\beta(X, \omega), X \in \mathfrak{C}$, is a random function that does not depend on events from $\sigma$-algebra $\mathcal{F}_{\tau}$ and is $\mathcal{G}_{\tau}$-measurable.

In order to prove the theorem, we need to demonstrate that for all $0 \leq s \leq \tau \leq$ $\leq t \leq T$ and $A \in \mathfrak{D}$ the following equality is true

$$
\mathbf{P}\left\{u_{t}(s, \cdot, \phi) \in A \mid \mathcal{F}_{\tau}\right\}=\mathbf{P}\left\{\tau, u_{\tau}(s, \cdot, \phi), t, A\right\}
$$

where $\mathbf{P}\{\tau, u, t, A\}$ is defined from (27). For proof of (64) it is enough to show that for any real bounded Borel function $g: \mathfrak{C} \rightarrow \mathbb{R}$ we have

$$
\mathbf{E}\left(g\left(u_{t}(s, \cdot, \phi)\right) \mid \mathcal{F}_{\tau}\right)=\left.\mathbf{E} g\left(u_{t}(\tau, \cdot, \varphi)\right)\right|_{\varphi=u_{\tau}(s, \cdot, \phi)} .
$$

The proof of (65) is proved similarly to [4, theorem 3.8] with the help of (63), independence of $u_{t}(\tau, \cdot, \varphi)$ from $\sigma$-algebra $\mathcal{F}_{\tau}$ and its $\mathcal{F}_{\tau}$-measurability. The theorem is proved. 


\section{EXAMPLE}

Let consider a particular case of the problem $(1)-\left(1^{*}\right)$

$$
\begin{gathered}
d\left(u(t, x)+\int_{\mathbb{R}} b(t, x, \xi) u(t-h, \xi) d \xi\right)=\left(\partial_{x}^{2} u(t, x)+f(t, u(t-h, x), x)\right) d t \\
+\sigma(t, u(t-h, x), x) d W(t, x), 0<t \leq T, x \in \mathbb{R}, \\
u(t, x)=\phi(t, x), \quad-h \leq t \leq 0, x \in \mathbb{R}, h>0 .
\end{gathered}
$$

Equation (66) is an equation of neutral type with constant delay. It can be a mathematical model with delay in nonlocal consumption of resources or nonlocal stimulation of reproduction [19].

Let function $b(t, x, \xi)$ have the form

$$
b(t, x, \xi)=\varphi(t, \xi) \psi(x)
$$

where $\varphi$ is continuous in $t$, and the following conditions fulfill:

1) there exists $\varphi_{1}$ such that $|\varphi(t, \xi)| \leq \varphi_{1}(\xi), 0 \leq t \leq T, \xi \in \mathbb{R}$;

2) $\psi$ is twice continuously differentiable and has bounded on $\mathbb{R}$ the first and the second derivatives $\psi^{\prime}, \psi^{\prime \prime}$;

3) $\left\{\psi, \varphi_{1}\right\} \subset L_{1}(\mathbb{R}) \cap L_{2}(\mathbb{R}),\left\{\psi^{\prime}, \psi^{\prime \prime}\right\} \subset L_{2}(\mathbb{R})$.

Then the conditions from assumptions $(5)-(7)$ are valid. If standard conditions from assumptions (2) (examples of $f$ and $\sigma$ can be easily given) and (4) fulfill, and estimate (25) is true, then all conditions of theorem 9 are true.

\section{APPENDIX}

We now prove proposition 3. Let $\varphi \in L_{p}([0, T], H)$ and set $\psi=$ $=R_{\gamma} \varphi$. We can write

$$
\begin{aligned}
\psi(t)-\psi(s) & =\int_{s}^{t}(t-\tau)^{\gamma-1} S(t-\tau) \varphi(\tau) d \tau \\
& +\int_{0}^{s}\left((t-\tau)^{\gamma-1}-(s-\tau)^{\gamma-1}\right) S(t-\tau) \varphi(\tau) d \tau \\
& +\int_{0}^{s}(s-\tau)^{\gamma-1}(S(t-\tau)-S(s-\tau)) \varphi(\tau) d \tau
\end{aligned}
$$




$$
=K_{1}+K_{2}+K_{3}
$$

We will estimate each term in (A.1) separately.

Since the semigroup $\{S(t), t \geq 0\}$ is contractive, we obtain for $K_{1}$

$$
\begin{aligned}
\left\|K_{1}\right\|_{H} & \leq \int_{s}^{t}(t-\tau)^{\gamma-1}\|\varphi(\tau)\|_{H} d \tau \\
& \leq\left(\int_{s}^{t}(t-\tau)^{q(\gamma-1)} d \tau\right)^{\frac{1}{q}}\left(\int_{s}^{t}\|\varphi(\tau)\|_{H}^{p} d \tau\right)^{\frac{1}{p}},
\end{aligned}
$$

where $\frac{1}{p}+\frac{1}{q}=1$. Since $\varphi \in L_{p}([0, T], H)$, there exists a constant $M_{1}>0$ such that

$$
\left\|K_{1}\right\|_{H} \leq M_{1}(t-s)^{\frac{1}{q}+\gamma-1}=M_{1}(t-s)^{\gamma-\frac{1}{p}} .
$$

For $K_{2}$ in (A.1), taking into account the estimate $\|S(t)\| \leq 1$, in the same way as in $\left[3\right.$, proposition A.1.1, p. 307], we obtain validity for some $M_{2}>0$ of the following inequality

$$
\left\|K_{2}\right\|_{H} \leq M_{2}(t-s)^{\gamma-\frac{1}{q}} .
$$

It remains to estimate the last term in (A.1). We will use the theorem on a dominated convergence with an integrable majorant $(s-\tau)\|\varphi(\tau)\|_{H}, s \leq \tau \leq t \leq T$, and take into account that the expression $S(t-\tau) \varphi(\tau)$ is continuous in $t$ for any fixed $\tau$. As a result, we conclude that $\left\|K_{3}\right\|_{H}$ is tending to zero as $t \rightarrow s, t \geq s$. In order to prove that $\left\|K_{3}\right\|_{H}$ is tending to zero as $s \rightarrow t, s \leq t$, let write $K_{3}$ in the following way

$$
\int_{0}^{t} \eta(\tau)\left(\frac{t-\tau}{s-\tau}\right)^{1-\gamma}(t-\tau)^{\gamma-1}(S(t-\tau)-S(s-\tau)) \varphi(\tau) d \tau,
$$

where $\eta(\tau)$ is the characteristic function of the time interval $[0, s]$. It is obvious that $0 \leq \tau \leq s$

for any $0 \leq \tau<t \underset{0 \leq \tau \leq s}{\eta(\tau)} \rightarrow \underset{0 \leq \tau \leq t}{\eta(\tau)}$ as $s \rightarrow t$, and $\frac{t-\tau}{s-\tau}$ is bounded as $s \rightarrow t$. Thus, using the theorem on a dominated convergence, we finish the proof of proposition 3.

\section{REFERENCES}

[1] V. Capasso, D. Fortunato, Stability results for semilinear evolution equations and their application to some reaction-diffusion problems, SIAM J. Math. Anal., 39 (1980), 37-47.

[2] Ye. F. Carkov, Sluchajnye Vozmusheniya Differencial'no-Funkcionalnyh Uravneny [Random Perturbations of Functional Differential Equations] (in Russian), Zinatne, Riga, 1989. 
[3] G. Da Prato, J. Zabczyk, Ergodicity for infinite dimensional systems, London Mathematical Society Lecture Note Series, 229, Cambridge University Press, (1996).

[4] G. Da Prato, J. Zabczyk, Stochastic equations in infinite dimensions, Encyclopedia of Mathematics and its Applications, Vol. 45, Cambridge University Press, 1992 .

[5] D. A. Dawson, Stochastic Evolution Equations, Math. Biosci., 15 (1972), 287316.

[6] Y. Du, S. Hsu, On a nonlocal reaction-diffusion problem arising from the modeling of phytoplankton growth, SIAM J. Math. Anal., 42 (2010), 1305-1333.

[7] L. C. Evans, Partial Differential Equations, American Mathematical Society, Providence, R. I., 1998.

[8] J. K. Hale, Theory of Functional Differential Equations, Applied Mathematical Sciences, Vol. 3, Springer-Verlag, New York, 1977.

[9] K. Ito, M. Nisio, On stationary solutions of a stochastic differential equation, 4 (1964), 1-75.

[10] A. F. Ivanov, Y. I. Kazmerchuk, A. V. Swishchuk, Theory, stochastic stability and applications of stochastic delay differential equations: a survey of recent results, Diff. Equ. and Dynam. Systems, 11 (2003), 55-115.

[11] V. B. Kolmanovskii, L. E. Shaikhet, Upravleniye Sistemami s Posledejstviyem [Control of Systems with After-Effect] (in Russian), Trans. Amer. Math. Soc., Providence, R. I., 157, 1996.

[12] V. B. Kolmanovskii, V. M. Nosov, Ustojchivost' i Periodicheskiye Rezhimy Reguliruyemykh Sistem s Posledejstviyem [Stability and Periodic Models of Control Systems with After-Effect] (in Russian), Nauka, Moscow, 1981.

[13] O. A. Ladyzhenskaya, V. A. Solonnikov, N. N. Ural'tseva, Linejnye i KvaziLinejnye Uravneniya Parabolicheskogo Tipa [Linear and Quasi-Linear Equations of Parabolic Type] (in Russian), Trans. Amer. Math. Soc., Providence, R. I., 23, 1968.

[14] N. I. Mahmudov, Existence and uniqueness results for neutral SDEs in Hilbert spaces, Stochastic Anal. Appl., 24 (2006), 79-95.

[15] X. Mao, Asymptotic properties of neutral stochastic differential delay equations, Stochastics Rep., 68 (2000), 273-295.

[16] X. Mao, Exponential stability in mean square of neutral stochastic differential difference equations, Dynam. Contin. Discrete Impuls. Systems, 6 (1999), 569586 . 
[17] X. Mao, A. Rodkina, N. Koroleva, Razumikhin-type theorems for neutral stochastic functional differential equations, Funct. Differ. Equ., 5 (1998), 195-211.

[18] H. P. McKean, Nagumo's equation, Advances in Math., 4 (1970), 209-223.

[19] V. Volpert, Elliptic Partial Differential Equations, Monographs in Mathematics, Vol. 104, Birkhäuser, Springer-Basel, 2014.

[20] K. Yosida, Functional Analysis, Grundlehren der Mathematischen Wissenschaften [Fundamental Principles of Mathematical Sciences], Vol. 123, Springer-Verlag, Berlin-New York, 1980. 